UPR-656-T

hep-ph/9504418

April, 1995

\title{
Improved Action Method for Analyzing Tunneling in Quantum Field Theory
}

\author{
Alexander Kusenko円 \\ Department of Physics and Astronomy \\ University of Pennsylvania \\ Philadelphia, PA 19104-6396
}

\begin{abstract}
We describe a new method which allows one to evaluate the false vacuum decay rate for a general potential which may depend on an arbitrary number of scalar fields.
\end{abstract}

* email address: sasha@langacker.hep.upenn.edu 


\section{Introduction}

The problem of calculating the transition probability between two inequivalent vacua in quantum field theory arises in different areas of high-energy physics, cosmology, and condensed matter physics. The semiclassical calculation of the false vacuum decay width was done in Refs. [1, 2, 3] for the case of a single scalar field, $\phi(x)$. The corresponding path integral is dominated by the field configuration $\bar{\phi}(x)$ called the "bounce" and can be evaluated using the saddle point method [2, 3]. The bounce, being the stationary point of the Euclidean action, is the non-trivial solution of the corresponding Euler-Lagrange equation which obeys certain boundary conditions.

Then the transition probability per unit volume, in the semiclassical limit [3] is

$$
\Gamma / \mathrm{V}=\frac{1}{h^{2}}(S[\bar{\phi}])^{2} e^{-S[\bar{\phi}] / \hbar}\left|\frac{\operatorname{det}^{\prime}\left[-\partial_{\mu}^{2}+U^{\prime \prime}(\bar{\phi})\right]}{\operatorname{det}\left[-\partial_{\mu}^{2}+U^{\prime \prime}(0)\right]}\right|^{-1 / 2} \times(1+O(\hbar))
$$

where $S[\bar{\phi}]$ is the Euclidean action of the bounce, and det' stands for the determinant with the zero eigenvalues omitted.

However, a number of practical applications require a generalization of this method to the case that involves several scalar fields. Although, from a theoretical point of view, such a generalization is rather straightforward円, in practice it is impossible to find the bounce except in trivial cases. The problem is that the bounce is an unstable solution of some system of non-linear differential equations which cannot be solved analytically. Small changes in the initial conditions lead to significant changes in the shape of the solution. In the case of a single scalar field, $\phi(x)$, one can find the bounce numerically by imposing the boundary condition on the value of the so called "escape point", $\phi_{e}=\phi(0)$, and constructing the "overshoot" and the "undershoot" solutions which envelope the bounce. Due to the special topology of the one-dimensional problem, one can then constrain the bounce to any given accuracy by varying the "escape" point, the value of the field at the center of the bounce, so that the "overshoot" and the "undershoot" solutions converge. If, however, the scalar

\footnotetext{
${ }^{1}$ If the potential has a continuum of degenerate minima, then the determinant in equation (1) acquires some additional zero modes, so that the right-hand side of (11) is, in fact, divergent. The false vaccum decay rate in this case was calculated in Ref. [4]
} 
field has $n>1$ components, this approach fails because an arbitrary path connecting the $\phi_{e}$ of the "overshoot" with that of the "undershoot" does not have to go through the "true" escape point.

The bounce $\bar{\phi}(x)$ is defined as a stationary point of the action $S[\phi]$ and is the solution of the variational equation $\delta S[\phi]=0$. However, it is not a minimum of $S[\phi]$ but rather a saddle point ‡.

If one could find some functional $\tilde{S}[\phi]$ for which the bounce would be a minimum, then one could define the the function $\phi(x)$ on a lattice and minimize $\tilde{S}[\phi]$ with respect to random variations of $\phi(x)$. This is the basic idea of our method. We will show that by adding some auxiliary terms to the action one can turn the saddle point into a true minimum. First, we derive some useful identities which help identify the terms that may be added to the action. And then we prove that the saddle point of the original action is a minimum of the improved action.

\section{General properties of the bounce}

Let us consider a quantum field theory with the scalar potential potential $U\left(\phi_{1}, \ldots, \phi_{n}\right)$ which has a local minimum at $\phi_{1}=\phi_{2}=\ldots=\phi_{n}=0, U(0, \ldots, 0)=0$ as well as at least one additional (local, or global) minimum at $\phi_{i}=\phi_{i}^{e}, i=1,2, \ldots, n ; U\left(\phi_{1}^{e}, \ldots, \phi_{n}^{e}\right)<0$. In the semiclassical limit [1, 2, 3], the transition probability is proportional to $\exp (-S[\bar{\phi}(x)])$, where $\bar{\phi}(x)=\left(\bar{\phi}_{1}(x), \ldots, \bar{\phi}_{n}(x)\right)$ is the so called "bounce", a nontrivial $O(4)$-symmetric [7] field configuration determined by the system of equations

$$
\Delta \bar{\phi}_{i}(r)=\frac{\partial}{\partial \bar{\phi}_{i}} U\left(\bar{\phi}_{1}, \ldots, \bar{\phi}_{n}\right)
$$

with the following boundary conditions:

\footnotetext{
2 The method proposed in Ref. [5] relies on the minimization of the action on the lattice with respect to the variations of the bounce profile, while maximizing the same action with respect to one particular variation, namely the scale transformation. Since the scaling corresponds to the direction of the instability of the bounce [6, 7], one can argue that it is desirable to minimize the action with respect to the variations orthogonal to scaling, and at the same time maximize it with respect to scaling. However, in practice, it is nearly impossible to identify the variations orthogonal to that related to scaling. And, in general, an iterative procedure of this kind does not converge to any limiting point.
} 


$$
\left\{\begin{array}{l}
\left.(d / d r) \bar{\phi}_{i}(r)\right|_{r=0}=0 \\
\bar{\phi}_{i}(\infty)=0
\end{array}\right.
$$

As was explained in the introduction, the chaotic instability of the solution of (2) 3ith with respect to small perturbations makes it impossible to solve the equation numerically. We therefore turn to the variational equation (for which (2) is the consecutive Euler-Langrange equation):

$$
\delta S=0
$$

where $S$ is the Euclidean action for a scalar field $\phi(x)$ :

$$
\begin{aligned}
S & =T+V \\
T & =\int d^{4} x \sum_{i=1}^{4} \frac{1}{2}\left(\frac{\partial \phi(x)}{\partial x_{i}}\right)^{2} \\
V & =\int U(\phi(x)) d^{4} x
\end{aligned}
$$

For any renormalizable theory ${ }^{3}$, the potential term in the Euclidean action $V=\int U(x) d^{4} x$ can be written as

$$
\begin{aligned}
V & =V_{2}+V_{3}+V_{4} \\
V_{n}[\phi] & =\int U_{n}(\phi(x)) d^{4} x
\end{aligned}
$$

where $U_{n}(\phi)$ is $n$-linear in $\phi$. In other words,

$$
V_{n}[\lambda \phi]=\int U_{n}(\lambda \phi(x)) d^{4} x=\int \lambda^{n} U_{n}(\phi(x)) d^{4} x=\lambda^{n} V_{n}[\phi]
$$

\footnotetext{
${ }^{3}$ In the presence of some higher-order (non-renormilizable) terms, equations (23) and (32) are modified accordingly to include the monomials of higher powers. However, the same method can be used to construct the improved action.
} 
Here we used the fact that $\phi=0$ is the local minimum and therefore no term, linear in $\phi$, is present in the potential.

By virtue of (4), the solution $\bar{\phi}(x)$ is the stationary point of the action. Therefore, for any family of functions $\phi_{\lambda}(x)$ such that $\left.\phi_{\lambda}(x)\right|_{\lambda=1}=\bar{\phi}(x)$, the corresponding action $S\left[\phi_{\lambda}(x)\right]$ should have a zero derivative with respect to $\lambda$ at $\lambda=1$ :

$$
\left.\frac{d}{d \lambda} S\left[\phi_{\lambda}(x)\right]\right|_{\lambda=1}=0
$$

In particular, one can choose

$$
\phi^{(\lambda)}(x)=\lambda^{p} \bar{\phi}\left(\lambda^{q} x\right)
$$

where $p$ and $q$ are arbitrary real numbers.

Then

$$
S\left[\phi^{(\lambda)}(x)\right]=\lambda^{2(p-q)} T+\sum_{n=2}^{4} \lambda^{p n-4 q} V_{n}
$$

The equation (11) implies

$$
\begin{aligned}
2[p-q] T+\sum_{n=2}^{4}[n p-4 q] V_{n} & =0 \Rightarrow \\
p\left(2 T+2 V_{2}+3 V_{3}+4 V_{4}\right)-2 q(T+2 V) & =0
\end{aligned}
$$

The relation (14) can be satisfied for all $p$ and $q$ if and only if

$$
\begin{aligned}
T+2 V & =0 \\
2 V_{2}+V_{3} & =0
\end{aligned}
$$

The first of these two identities (15) is well known and can be derived [6, 7] using the variation of the type (12) with $p=0, q=1$. 
Another useful identity can be obtained by considering the following variation about the bounce:

$$
\left\{\begin{array}{l}
\bar{\phi}_{i}(x) \rightarrow \phi_{i}^{(\lambda)}(x)=\bar{\phi}_{i}(x)-\lambda \bar{\phi}_{j}(x) \\
\bar{\phi}_{j}(x) \rightarrow \phi_{j}^{(\lambda)}(x)=\bar{\phi}_{j}(x)+\lambda \bar{\phi}_{i}(x) \\
\bar{\phi}_{k}(x) \rightarrow \phi_{k}^{(\lambda)}(x)=\bar{\phi}_{k}(x), \quad k \neq i, j
\end{array}\right.
$$

It is convenient to write $(17)$ as

$$
z(x) \equiv \bar{\phi}_{i}(x)+i \bar{\phi}_{j}(x) \rightarrow z^{(\lambda)}(x)=e^{i \lambda}\left(\bar{\phi}_{i}(x)+i \bar{\phi}_{j}(x)\right)
$$

Then it is clear that the kinetic term in the Euclidean action

$$
\begin{array}{r}
T=\int d^{4} x \sum_{a, k}\left(\frac{d \phi_{k}\left(x_{a}\right)}{d x_{a}}\right)^{2}= \\
\int d^{4} x \sum_{a ; k \neq i, j}\left(\frac{d \phi_{k}\left(x_{a}\right)}{d x_{a}}\right)^{2}+\sum_{a}\left(\frac{d}{d x_{a}} \bar{z}\right)\left(\frac{d}{d x_{a}} z\right)
\end{array}
$$

is invariant under the transformation (18), and therefore under the transformation (17).

It is also clear that the transformation (17) preserves the boundary conditions (3). In other words, if the constraints (3) are satisfied for $\lambda=0$, then they are also satisfied for any non-zero value of $\lambda$.

We now apply the transformation (17) to the Euclidean action and require that it have zero derivative with respect to the parameter $\lambda$ at $\lambda=0$ :

$$
\begin{array}{r}
0=(d / d \lambda) S\left[\phi_{\lambda}\right]_{\lambda=0}=(d / d \lambda)(T+V)_{\lambda=0}=\left.(d / d \lambda) V\right|_{\lambda=0}= \\
\left.\int d^{4} x\left(\phi_{j}(x) \frac{\partial}{\partial \phi_{i}}-\phi_{i}(x) \frac{\partial}{\partial \phi_{j}}\right) U(\phi)\right|_{\phi(x)=\bar{\phi}(x)}
\end{array}
$$

Thus for an arbitrary pair of $\phi_{i}$ and $\phi_{j}$ we obtain the constraint:

$$
\left.\int d^{4} x \operatorname{det}\left|\begin{array}{cc}
\frac{\partial}{\partial \phi_{i}} & \frac{\partial}{\partial \phi_{j}} \\
\phi_{i} & \phi_{j}
\end{array}\right| U\left(\phi_{1}, \ldots, \phi_{n}\right)\right|_{\phi(x)=\bar{\phi}(x)}=0
$$


There are $(n-1)$ linearly-independent constraints of the form (21) for $(n-1)$ pairs of components of the $n$-component field $\phi$.

\section{Improved Action method}

In the preceeding section we proved that the following quantities vanish identically:

$$
\begin{aligned}
\Lambda_{1} & =T[\bar{\phi}(x)]+2 V[\bar{\phi}(x)] \\
\Lambda_{2} & =2 V_{2}[\bar{\phi}(x)]+V_{3}[\bar{\phi}(x)] \\
\Lambda_{3}^{i j} & =\left.\int d^{4} x \operatorname{det}\left|\begin{array}{cc}
\frac{\partial}{\partial \phi_{i}} & \frac{\partial}{\partial \phi_{j}} \\
\phi_{i} & \phi_{j}
\end{array}\right| U\left(\phi_{1}, \ldots, \phi_{n}\right)\right|_{\phi(x)=\bar{\phi}(x)}
\end{aligned}
$$

In order to determine the bounce numerically, we would like to find some functional $\tilde{S}[\phi]$ for which the bounce $\bar{\phi}(x)$ would be a minimum. Then one can discretize the problem by defining the field $\phi(x)$ on a lattice. The improved action, $\tilde{S}[\phi]$ can then be minimized with respect to random perturbations of the field $\phi$ until the limiting bounce is recovered to a required accuracył.

We define $\tilde{S}[\phi]$ as the action $S[\phi]$ plus some auxiliary terms which would vanish as $\phi(x)$ approaches $\bar{\phi}(x)$ :

$$
\tilde{S}[\phi]:=S[\phi]+\sum_{n}\left(\alpha_{1, n}\left|\Lambda_{1}\right|^{p_{n}}+\alpha_{2, n}\left|\Lambda_{2}\right|^{p_{n}}+\alpha_{3, i j, n}\left|\Lambda_{3}^{i j}\right|^{p_{n}}\right),
$$

where $p_{n}, n=1,2, \ldots$ are some positive numbers, and $\alpha_{n}>0$ are some arbitrary Lagrange multipliers.

In particular, we will show that it suffices to take

$$
\tilde{S}[\phi]:=S[\phi]+\alpha_{1}\left|\Lambda_{1}\right|
$$

\footnotetext{
${ }^{4}$ Clearly, it is not even necessary that $\tilde{S}[\phi]$ be differentiable with respect to $\phi$ at $\phi=\bar{\phi}(x)$.
} 
but in practice one can include other terms as well to speed up the convergence of the iterative routine.

Now we want to prove the following statement:

Theorem 1. If $\delta S[\bar{\phi}(x)]=0$, then $\bar{\phi}(x)$ is a local minimum of the functional $\tilde{S}[\phi(x)]$.

Proof. By construction, the difference $\tilde{S}[\phi]-S[\phi]=\alpha_{1}\left|\Lambda_{1}\right| \geq 0$, with equality possible only if $\Lambda_{1}[\phi]=0$. Consider some family of functions $\phi_{\lambda}(x), 0<\lambda<2$ such that $\left.\phi_{\lambda}(x)\right|_{\lambda=1}=\bar{\phi}(x)$. Then

$$
\left.\frac{d}{d \lambda} \tilde{S}\left[\phi_{\lambda}(x)\right]\right|_{\lambda=1}=\alpha_{1} \frac{d}{d \lambda}\left|\Lambda_{1}\left[\phi^{(\lambda)}(x)\right]\right|
$$

because $\frac{d}{d \lambda} S\left[\phi_{\lambda}(x)\right]=0$ at $\lambda=1$.

Consider the following two cases.

1) Suppose $\frac{d}{d \lambda} \Lambda_{1}\left[\phi^{(\lambda)}(x)\right] \neq 0$. Since $\left|\Lambda_{1}\left[\phi^{(\lambda)}(x)\right]\right| \geq 0,\left|\Lambda_{1}[\bar{\phi}(x)]\right|=\inf \left|\Lambda_{1}\left[\phi^{(\lambda)}(x)\right]\right|=0$. Therefore, if $\frac{d}{d \lambda}\left|\Lambda_{1}\left[\phi^{(\lambda)}(x)\right]\right| \neq 0$, then $\bar{\phi}(x)$ is a local minimum of $\tilde{S}\left[\phi^{(\lambda)}(x)\right]$ with respect to $\lambda$.

2) If, on the other hand, $\frac{d}{d \lambda}\left|\Lambda_{1}\left[\phi^{(\lambda)}(x)\right]\right|_{\lambda=1}=0$, then

$$
\begin{array}{r}
0=\frac{d}{d \lambda} \Lambda_{1}\left[\phi^{(\lambda)}(x)\right]_{\lambda=1}=\left.\frac{d}{d \lambda}\left(T\left[\phi^{(\lambda)}(x)\right]+2 V\left[\phi^{(\lambda)}(x)\right]\right)\right|_{\lambda=1}= \\
\left.\frac{d}{d \lambda}\left(S\left[\phi^{(\lambda)}(x)\right]+V\left[\phi^{(\lambda)}(x)\right]\right)\right|_{\lambda=1}=\frac{d}{d \lambda} V\left[\phi^{(\lambda)}(x)\right]_{\lambda=1}
\end{array}
$$

or, in other words, $\Lambda_{1}$ can have a zero derivative only in the direction of the constant $V$. However, it was shown in Ref. [7] that the bounce $\bar{\phi}(x)$ is the global minimum of the action $S[\phi]$ over the surface of constant $V$.

In other words, the variation $\left(\frac{d}{d \lambda} \phi^{(\lambda)}(x)\right) d \lambda$ is tangential to the surface $V=$ const, while the only [7] eigenvector of the second derivative operator $\left(\delta^{2} S / \delta \phi_{i} \delta \phi_{j}\right)=\delta_{i j} \Delta+$ $\left(\partial^{2} U(x) / \partial \phi_{i} \partial \phi_{j}\right)$ which corresponds to a negative eigenvalue was shown in Ref. [7] to be orthogonal to the surface $V=V[\bar{\phi}]=$ const in the functional space. Therefore, $\bar{\phi}$ is a minimum of $\tilde{S}$ in this case as well. 
We have shown that for an arbitrary variation $\phi^{(\lambda)}(x)$ near the bounce $\bar{\phi}(x)$, either (i) $\delta S=0, \delta\left|\Lambda_{1}\right|^{p}>0$, or (ii) $\delta S=\delta \Lambda_{1}=0$, but $\delta^{2} S / \delta \phi^{2}$ is positive definite. In either case, $\bar{\phi}$ is the minimum of $\tilde{S}$. This completes the proof.

Therefore, the improved action (26), as well as a more general form (25) has the desired solution $\bar{\phi}(x)$ as its minimum. This justifies the method described above of searching for the bounce as a minimum of the improved action rather than a saddle point of the actual action.

Our method can be easily generalized to the case of finite-temperature field theory. In the high-temperature limit, the transition probability [8],

$$
\Gamma / \mathrm{V}=T\left(\frac{S_{3}[\tilde{\phi}, T]}{h T}\right)^{3 / 2} e^{-S_{3}[\tilde{\phi}, T] / \hbar T}\left|\frac{\operatorname{det}^{\prime}\left[-\partial_{\mu}^{2}+U^{\prime \prime}(\tilde{\phi}, T)\right]}{\operatorname{det}\left[-\partial_{\mu}^{2}+U^{\prime \prime}(0, T)\right]}\right|^{-1 / 2} \times(1+O(\hbar)),
$$

is determined by the three-dimensional action $S_{3}(\tilde{\phi}, T)$ of the $\mathrm{O}(3)$-symmetric solution $\tilde{\phi}(x)$ of the equation $\delta S_{3}=0$. As before, $\tilde{\phi}(x)$ is a saddle point of $S_{3}[\phi]$. The improved action $\tilde{S}_{3}[\phi]$, for which $\tilde{\phi}(x)$ is a minimum, can be constructed by analogy with equation (25):

$$
\tilde{S}_{3}[\phi]:=S_{3}[\phi]+\sum_{n}\left(\alpha_{1, n}\left|\Lambda_{1}^{(T)}\right|^{p_{n}}+\alpha_{2, n}\left|\Lambda_{2}^{(T)}\right|^{p_{n}}+\alpha_{3, i j, n}\left|\Lambda_{3}^{(T) i j}\right|^{p_{n}}\right) ; \quad p_{n}, \alpha_{n}>0
$$

where

$$
\begin{aligned}
\Lambda_{1}^{(T)} & =T+3 V \\
\Lambda_{2}^{(T)} & =\sum_{n}(6-n) V_{n} \\
\Lambda_{3}^{(T) i j} & =\left.\int d^{3} x \operatorname{det}\left|\begin{array}{cc}
\frac{\partial}{\partial \phi_{i}} & \frac{\partial}{\partial \phi_{j}} \\
\phi_{i} & \phi_{j}
\end{array}\right| U\left(\phi_{1}, \ldots, \phi_{n}, T\right)\right|_{\phi(x)=\bar{\phi}(x)}
\end{aligned}
$$

The application of our method to the issue of stability of the charge and color conserving vacuum in the MSSM will be presented in [9].

The author would like to thank P. Langacker, A. Linde, G. Segre and P. Steinhardt for stimulating discussions. This work was supported by the U. S. Department of Energy Contract No. DE-AC02-76-ERO-3071. 


\section{References}

[1] M. B. Voloshin, I. Yu. Kobzarev and L. B. Okun', Yad. Fiz. 20 (1974) 1229 [Sov. J. Nucl. Phys. 20 (1975) 644 ].

[2] S. Coleman, Phys. Rev. D15 (1977) 2929.

[3] C. G. Callan and S. Coleman, Phys. Rev. D16 (1977) 1762.

[4] A. Kusenko, University of Pennsylvania preprint UPR-671-T (hep-ph/9506386).

[5] M. Claudson, L. J. Hall and I. Hinchliffe, Nucl. Phys. B228 (1983) 501.

[6] G. H. Derrick, J. Math Phys. 5 (1964) 1252.

[7] S. Coleman, V. Glaser and A. Martin, Comm Math. Phys. 58 (1978) 211.

[8] A. D. Linde, Phys. Lett B70 (1977) 306; ibid. B100 (1981) 37; Nucl. Phys. B216 (1983) 421.

[9] A. Kusenko, P. Langacker and G. Segre, work in progress. 